\title{
Functional Integration of Human-Like Localization in a Decision Unit for Autonomous Agents
}

\author{
Christian Brandstaetter, Klaus Doblhammer, Martin Fittner
}

\begin{abstract}
Localization is from the purely technical point of view an already solved problem. There are working selflocalization algorithms and a lot of very good navigation algorithms. The aim of this paper is a little bit different. We want to identify the way, how humans do localization. How humans localize themselves and find their way to desired objects. Psychoanalysis is the cornerstone we get our insight into the human brain from. Using this different approach, we will not beat existing navigation systems in speed or performance. This is not our target. What we like to do is, to support psychoanalysis in verifying their theory by implementing a model of the human mind in a technical simulation. In the paper at hand, we describe where the different methods of self-localization and navigation are located within the whole model.
\end{abstract}

Keywords - artificial recognition system, bionic, localization, navigation, psychoanalysis

\section{Introduction}

In the year 2000, the project Artificial Recognition System (ARS) was founded at the Institute of Computer Technology (ICT) at Vienna University of Technology by Dietmar Dietrich and his colleague Thilo Sauter [1]. Since that time a team of engineers is working on developing a model of the human brain. Verification is done by implementing the model in a multi-agent simulation. The original plan was to create an intelligent system which can recognize and understand situations in real-world. For example, the potential risk of hazards in a kitchen. It became clear that the way human beings perform recognition tasks is by using feelings. The theory on which this project is constructed on is psychoanalysis because this science provides concepts of how the mind works. Psychoanalysis is not a strict natural science. So, we, as technicians, will not be able to interpret it directly. To be able to use it anyway, psychoanalytical advisors have joined the project team. The name Artificial Recognition System (ARS) was not suitable anymore and therefore changed to Simulation of Mental Apparatus and Applications (SiMA). The SiMA model is based on the structural model or second topographical model of psychoanalysis [2] and incorporates the Id, Ego, and Super-ego as major parts. There are unconscious and conscious parts which implies restrictions on the way data can get processed. Not all the data is available everywhere. The results of localization can be inaccurate. The internal belief of the agent can derivate from its absolute position.

Christian Brandstaetter, Klaus Doblhammer, Martin Fittner Institute of Computer Technology at Vienna University of Technology Gußhausstraße 27-29, 1040 Vienna, Austria
This is similar to the subjective sense of time. Depending on various aspects, we can heavily over- or underestimate the length of an experienced timespan.

\section{The SiMA Model}

During the first decade of the project SiMA the model evolved to the depicted shape (Fig. 1). It was developed together with the psychoanalytical advisors in a top-down design method.

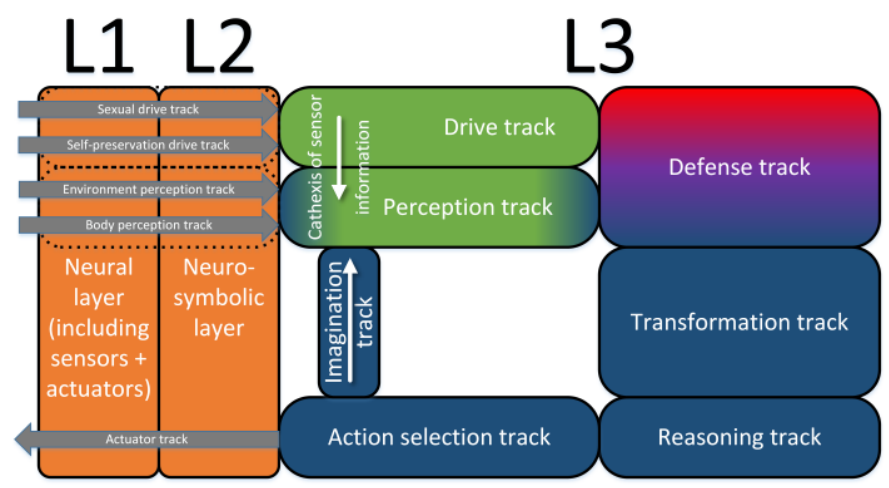

Fig. 1. High-Level representation of the SiMA Model, published in [3]

The model consists of three Layers (L1, L2, and L3 from left to right). The layer L1 represents the hardware of the model. It is connected to the physical, non-neuronal body. In the upper half of the model data enters the model and in the lower half information is passed on to various actuators. The layer L2 is the neurosymbolization layer. It is the link between body and psyche and was introduced by Rosemarie Velik in [4]. The layer L3 is the Psyche itself. It is the major part, where research is going on during the last years within SiMA. Let us explain the different tracks in the following paragraph.

In the drive track drive impulses from the sexual drive track, and the self-preservation track originates in the body and are represented as symbols. In the perception track, perception contents from the environment and from inside the body come to the psyche and get manipulated individually. The perception track gets additional information from the drive track (cathexis of sensor information). The various psychoanalytical called id-contents come into conflict with each other and with the super-ego demands. To resolve this, the ego suppresses or alters some content by using defense mechanisms, located in the defense track. Id-contents is labeled in Fig. 1 in green color, super-ego demands are red, and the ego is blue. In the transformation track, the contents are passed on from the upper primary process to the lower secondary process. Content gets consciousness-capable and is now compatible with the temporal and rational organization. 
In the reasoning track, a pre-conscious focusing on the particular need or wish is done. In the action selection track, the action to be used is chosen and brought into focus. In the imagination, track imagined actions are transmitted to the primary process and affect perception there. In the action track, the intentions are passed on to the body for execution.

\section{How to add Localization to the SiMA model}

As the aim of the project SiMA is to translate the theory of psychoanalysis into a model of natural science, the separation into the primary and secondary process is of high importance. The major part of the intelligence is done in the primary process where thoughts are unconscious. As a preliminary work of the paper at hand, we have outlined in [5] how human inspired navigation can work for simulated agents. After further discussion in the project team, it became clear that the primary process has for sure influence on the navigation process. This influence was investigated in [6] in detail. Now, as there is a stable view of how human-like navigation should work, we have aligned the described navigation- and selflocalization-methods to the functional modules of the SiMA model. This model of the project SiMA is based on Fig. 1 and shows in a detailed diagram the flow of data between the particular functional modules. While a description of the whole model, which can be found at the Projects website (http://sima.ict.tuwien.ac.at/ars-model/), goes beyond the scope of this paper, we would like to describe the functions, which contribute to the navigation process.

\section{A. Interaction between Primary- and Secondary-Process}

It turned out that there is a large impact of the primary process on self-localization and navigation. Important data, which originates in the primary process is the drive object, the current perception, and the current location ${ }^{1}$. This data is enriched in the primary process by associating locations ${ }^{2}$ and regions $^{3}$ to it. Depending on the Quota of Affect ${ }^{4}$ the agent can associate more or less deep in the network of remembered connections [7]. All the locations and regions get evaluated regarding how good they fit the current emotional situation of the agent. Before passing these data on to the secondary

\footnotetext{
${ }^{1}$ As part of the self-representation of the agent. The current location is finally determined in the secondary process and fed back to the primary process via the imagination track.

${ }^{2}$ locations are remembered places, defined by the landmarks which surround them

${ }^{3}$ regions hierarchical groups locations or regions together

${ }^{4}$ The Quota of Affect, as described in the Natural scientificpsychoanalytical glossary (NPyG, see appendix of [8]), is a potential, an evaluation-variable, which derives from a drive and represents an equivalent to drive-tension. It cathects conscious and unconscious contents and it can be displaced within unconscious mental mechanisms. The discharge of a quota of affect produces pleasure. Quotas of affect are the basic parameters of emotions and feelings.
}

process, the defense mechanisms modify it [9]. Things get removed, modified or completely replaced. So, in the primary process is all about associating memories with things currently in mind. This supports navigation as well as the selflocalization. For the self-localization, there is a virtual 360degree view generated in the primary process by stitching together landmarks from the vision over time while the agent moves and turns. This consolidated 360-degree view is used to determine the current location of the agent in the secondary process as described in [5]. The navigation is based on the associated trees from the primary process seeded by the current location and the drive object. When these two trees connect to each other, navigation is possible because these two trees form a connected network. In the secondary process, the best connection between current location and the drive object is determined by incorporating the available relations from the secondary process. As on top of the locations, there are multiple hierarchical layers of regions a deepest common region algorithm described in [5] is used, to truncate unneeded branches of the tree and focus just on relevant sections. It is possible to find the way from the current location and the drive object first in the much simpler high-level regions and afterward refine the path on lower levels. On the lowest level, containing the locations, the connections between them identify the actions to use for the navigation.

\section{B. Integrating the Functionality into the existing Model}

To describe the functionality aligned to the existing model we have unfolded the model in Fig. 2. The Information is basically flowing from top to bottom. The only exception here is the imagination track, which feeds information back from the secondary process to the primary process. The Figure is a combination of High-Level Track-View and detailed module diagram of the current SiMA model. It is more detailed, where modules are important for navigation and self-localization and do not show modules which are less relevant for navigation and self-localization, to keep the overview of the whole model. There are different presentations used:

- Light gray boxes with rounded corners correspond to the tracks in the model (see Fig. 1)

- Boxes and ellipses with blue filling are distinct modules from the model (Boxes have interfaces to fixed data and personality parameters, Ellipses do not have them)

- Light blue edges represent the high-level data flow between the different tracks (see Fig. 1)

- Black solid edges represent data flow between distinct modules

- Black dashed edges represent data flow between modules, but with hidden modules in-between, which are not shown because they are not important for navigation and self-localization regarding our examination. 


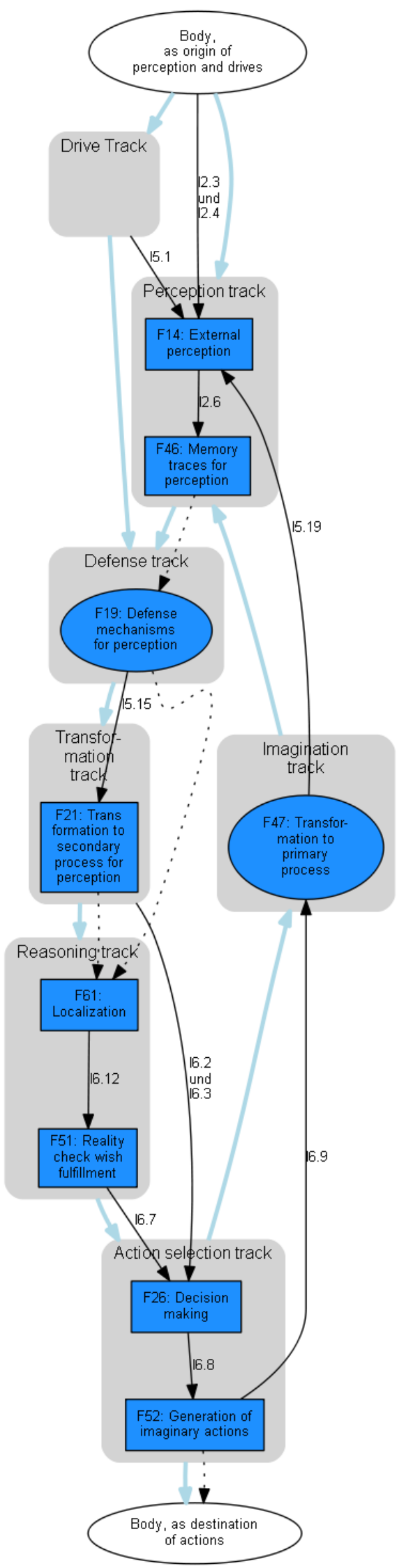

Fig. 2. Detailed view of the SiMA Model with regards to localization.
F14 External Perception aggregates drive object, the current perception, and the current location. The current perception originates in various sensors, the drive object comes from the drive track, and the current location is passed on from the secondary process via the imagination track.

F46 Memory traces associates locations and regions to them. Evaluates them regarding how good they fit the current emotional situation of the agent. Depending on the quota of affect, there get more or fewer locations and regions associated to drive object, the current perception, and the current location. The association process is similar to the spread activation described in [10, p. 61].

After F46 there are several functions in the model, before the data arrives at F19. But their functionality has no direct influence on the process of localization and navigation. F19 Defense mechanisms for perception modifies the network of the recalled locations and regions in case of a conflict. Depending on the super-ego, parts are removed, altered or modified [9].

F21 Transformation to the secondary process for perception translates locations, regions the perceived landmarks from unconscious to conscious objects. Relations like Left-of/Right-of and Nearer-than/Farer-than come from the perception and are now usable. The memory is searched for each pair of location to identify actions, which connects them to each other.

Other modules in the Transformation track convert emotions to feelings and pass the drive object on to the secondary process.

F61 Localization executes the self-localization based on the associated locations and the perceived landmarks and delivers the current location.

F51 Reality Check Wish Fulfilment has filtered out drive objects out of sight before adding localization to the model. Now, this is not necessary anymore. Even drive objects out of view are possible targets.

F52 Generation of imaginary actions determines the path from the current location to the drive object using the associated locations and regions in-between, if such a connection exists. When there are more than one points of contact between the associated trees of the current location to the drive object, the evaluation of the best path is influenced by the evaluation from F46. The detailed process of finding the path is described in [5].

F47 Transformation to primary process passes the current location on to the primary process. The current location is part of the self-representation of the agent.

The Action selection track delivers the next action to take, as a result of the navigation process. This action is forwarded to the body for execution.

\section{Extension of existing Data Types}

Up to now basically everything, the agent can perceive in the simulation, is of data type WPM:ENTITY. WPM stands for word presentation mesh and describes a complex of presentations associated towards each other, which refer to a word of (human) language. This includes e.g. sound pattern, 
character, typeface, moving image, etc. whereas, concerning the usual use of language in practice, the sound pattern prevails (NPyG, see appendix of [8]). There

WPM:LOCATION and WPM:REGION is added. The existing WPM:ENTITY is used for landmarks. The existing WPM:ACTION is used for actions, which connect locations to each other. All the possible associations are listed in Table 1.

\begin{tabular}{|c|c|c|}
\hline Object 1 & predicate & Object 2 \\
\hline Entity & is located in & Location \\
\hline Location & contains & Entity \\
\hline Location & has & Action \\
\hline Action & leads to & Location \\
\hline Action & originates at & Location \\
\hline Location & is located in & Region \\
\hline Region & contains & Location \\
\hline
\end{tabular}

Table 1. Associations between data types entity, location, and region.

Actions and Locations have associated memories with them. These memories cause the action or location to be more desirable or less desirable in different emotional situations of the agent. These memories are stored condensed as listed in Table 2.

\begin{tabular}{|c|c|c|c|}
\hline Object 1 & predicate & Object 2 & $\begin{array}{l}\text { Strength of } \\
\text { Association }\end{array}$ \\
\hline Action & satisfies & Anxiety & \multirow{8}{*}{$\begin{array}{l}\text { How desirable } \\
\text { this } \\
\text { action/location is } \\
\text { regarding the } \\
\text { current feeling }\end{array}$} \\
\hline Action & satisfies & Joy & \\
\hline Action & satisfies & Anger & \\
\hline Action & satisfies & Mourning & \\
\hline Location & satisfies & Anxiety & \\
\hline Location & satisfies & Joy & \\
\hline Location & satisfies & Anger & \\
\hline Location & satisfies & Mourning & \\
\hline
\end{tabular}

Table 2. Associations to different emotional situations

\section{Sketch of an Example}

Let us imagine an agent on a sightseeing tour in the beautiful city of Vienna. He starts his tour near the Karl's Place in the south marked with a red circle in the map (Fig. 3). He is required to finish his tour near the target at the Sweden Place in the north, marked with a green circle. It is now up to the agent to visit all attractions or just some of them. The lines between the attractions are possible ways, the agent can walk. Based on just rational reasons, the agent might use the direct connection via Stephan's Cathedral. But when taking memories and feelings into account, the agent might take a different way. For example, when the agent has special interest in academic buildings, he might visit the university. When the agent is sleepy, he might take a nap on a bench in the park before continuing his journey. It is possible that the agent changes his plan while on the way. Maybe he is hungry after starting his walk. So, the agent will visit the shops at the mall of Landstrasse first to get some food. After his hunger has decreased the rest of the way is evaluated under a different perspective and the agent might change his ongoing way.

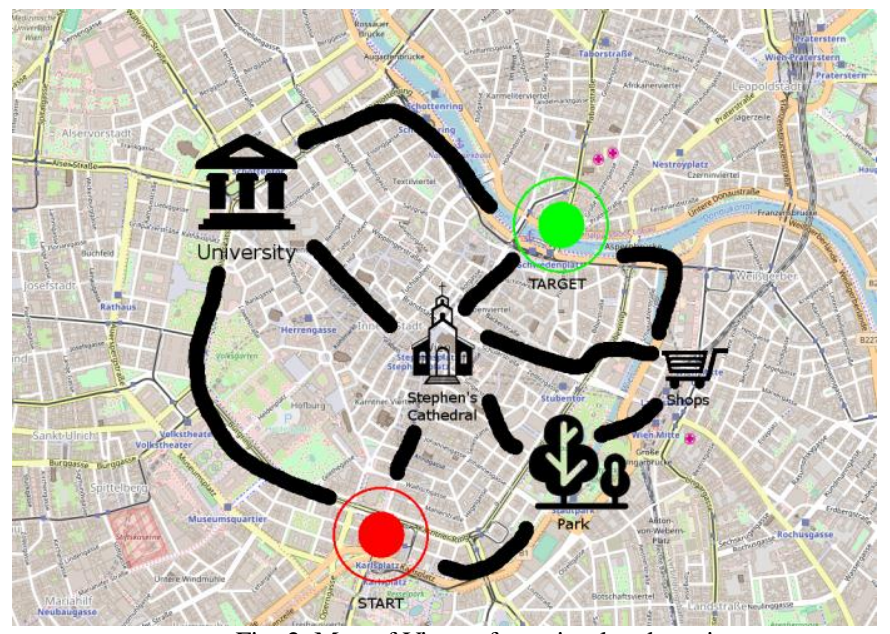

Fig. 2. Map of Vienna for a simulated tourist.

Let us give another example aside of the map of Vienna.

Sometimes car drivers are given the opportunity of taking a street over the mountain pass or a tunnel under the mountain. We think, this decision is heavily influenced by feelings. Is the agent interested in nice landscape and not so much in hurry, he might take the street over the mountain pass. This street might also satisfy the aggressive demands in sportive driving of an agent by providing lots of serpentines. Is the agent in hurry and in a relaxed mood, he might take the tunnel because of being faster.

This type of consideration for agent navigation might not lead to faster reaching the target. But it can help planning institutions of streets and public transportation to better understand how humans move by being motivated based on feelings.

\section{v. Conclusion}

It has turned out that developing human-inspired navigation and self-localization is just the halfway of having a simulated agent behave human-like. We continued to the investigation, how unconscious thinking influences the human navigation in [6], which lead us the work at hand. So, we have all the developed methods aligned with the existing model, ready for implementation. Stay tuned for the results of brain-like location awareness and navigation for psychoanalytically inspired autonomous agents in the simulation. 


\section{References}

[1] D00: Dietrich, Dietmar: Evolution potentials for Feldbus systems. In: Factory Communication Systems, 2000. Proceedings. 2000 IEEE International Workshop on Bd. 1, 2000, 145-146. - Invited Talk

[2] Fre23: Freud, Sigmund: The Ego and the Id. In: The Standard Edition of the Complete Psychological Works of Sigmund Freud XIX (1923-1925) (1923), S.1-66

[3] DJS+17 Dietrich, Dietmar ; Jakubec, Matthias ; Schaat, Samer ; Doblhammer, Klaus ; Fodor, Georg ; Brandstätter, Christian: The Fourth Outrage of Man - Is the Turing-test Still Up to Date? In: Journal of $\begin{array}{lllllll}\text { Computers } & 12 & \text { (2017), } & \text { Nr. } & 2, & 116 & -126 .\end{array}$ http://dx.doi.org/10.17706/jcp.12.2.116-126.

[4] Vel08: Velik, Rosemarie: A Bionic Model for Human-like Machine Perception, Vienna University of Technology, Institute of Computer Technology, Diss., 2008

[5] BSW+17 Brandstaetter, C., Schaat, S., Wendt, A., \& Fittner, M. (2017). How Agents use Breadcrumbs to Find their Way. Journal of Computers, 12(1), 89-96. http://doi.org/10.17706/jcp.12.1.89-96

[6] BF18 Brandstätter, C., \& Fittner, M. (2018). Enabling smart navigation by incorporating human inspired evaluation of route sections. Journal of Computers, 13(2), 187-193. http://doi.org/10.17706/jcp.13.2.187-193

[7] Lan10 Lang, R. (2010). A Decision Unit for Autonomous Agents Based on the Theory of Psychoanalysis. Vienna Univesity of Technology.

[8] BDD+15 Brandstätter, C., Dietrich, D., Doblhammer, K., Fittner, M., Fodor, G., Gelbard, F., ... Widholm, R. (2015). Natural Scientific, Psychoanalytical Model of the Psyche for Simulation and Emulation.

[9] Gel17 Gelbard, F. (2017). Psychoanalytic Defense Mechanisms in Cognitive Multi-Agent Systems. Taylor \& Francis.

[10] Wen16 Wendt, Alexander: Experience-Based Decision-Making in a Cognitive Architecture, Supervisor: D. Dietrich, Y. Biletsky; E384, 2016
About Author (s):

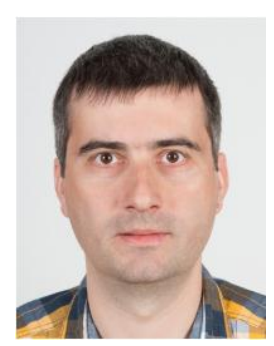

Christian Brandstätter was born 1979 at Tulln (Austria), studied Informatics and InformaticsManagement at the Vienna University of Technology and finished both studies with the degree Master of Science.

He works as System Administrator for general IT in a pharmaceutical research institute. His responsibility there is the IT infrastructure (network, storage, and security). Currently, he works on his dissertation and is a scientific member of the Institute of Computer Technology at the Vienna University of Technology. Brandstätter, M.Sc. is a member of IEEE.

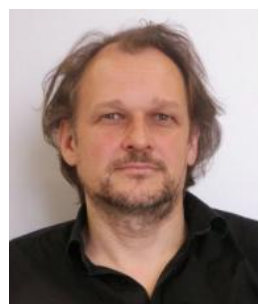

Klaus Doblhammer was born 1966 at Schärding (Austria), he studied physics then pedagogy at University of Vienna and finished with the degree of a Ph.D.

He worked as a supervisor for social-therapeutic institutions and was a scientific member of the Institute of Computer Technology, Vienna University of Technology from 2009 to 2015. Currently, Dr. Doblhammer is a psychoanalyst in Vienna and member of the Vienna Psychoanalytic

Association (WAP).

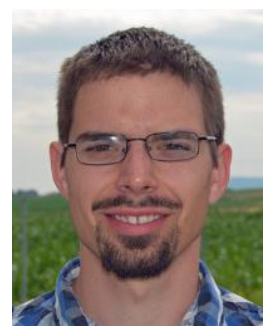

Martin Fittner was born 1984 at Mistelbach (Austria), studied Embedded Systems at the University of Applied Science Technikum Wien in Vienna and finished with the degree Master of Science.

He works as a senior developer for software and system design for automotive electronic systems at the Robert Bosch AG. He is a member of the SiMA (Simulation of the Mental Apparatus and Applications) team. Currently, he works on his dissertation at the Vienna University of Technology in the field of the SiMA project. 